\title{
Congenital hypogonadotropic hypogonadism with split hand/foot malformation: a clinical entity with a high frequency of FGFR1 mutations
}

\author{
Carine Villanueva, MD, PhD ${ }^{1,2}$, Elka Jacobson-Dickman, $\mathrm{MD}^{3,4}$, Cheng $\mathrm{Xu}, \mathrm{MD}^{5-7}$, \\ Sylvie Manouvrier, MD, $\mathrm{PhD}^{8}$, Andrew A. Dwyer, PhD ${ }^{3,6,7}$, Gerasimos P. Sykiotis, MD, PhD ${ }^{3,6,7}$, \\ Andrew Beenken, MD, PhD ${ }^{9}$, Yang Liu, MSc9 ${ }^{9}$ Johanna Tommiska, PhD ${ }^{10}$, Youli Hu, MD, PhD ${ }^{11}$, \\ Dov Tiosano, MD ${ }^{12,13}$, Marion Gerard, MD ${ }^{14}$, Juliane Leger, MD ${ }^{12,15}$, Valérie Drouin-Garraud, MD ${ }^{16}$, \\ Hervé Lefebvre, MD, PhD ${ }^{17}$, Michel Polak, MD, PhD ${ }^{18}$, Jean-Claude Carel, MD, PhD ${ }^{1,2,15}$, \\ Franziska Phan-Hug, MD ${ }^{19}$, Michael Hauschild, MD ${ }^{19}$, Lacey Plummer, BS ${ }^{3}$, Jean-Pierre Rey, BS ${ }^{6,7}$, \\ Taneli Raivio, MD, PhD ${ }^{10}$, Pierre Bouloux, MD ${ }^{11}$, Yisrael Sidis, PhD ${ }^{3,6,7}$, Moosa Mohammadi, PhD ${ }^{9}$, \\ Nicolas de Roux, MD, PhD ${ }^{1,2,20}$ and Nelly Pitteloud, MD ${ }^{3,6,7}$
}

Purpose: Congenital hypogonadotropic hypogonadism $(\mathrm{CHH})$ and split hand/foot malformation (SHFM) are two rare genetic conditions. Here we report a clinical entity comprising the two.

Methods: We identified patients with $\mathrm{CHH}$ and SHFM through international collaboration. Probands and available family members underwent phenotyping and screening for FGFR1 mutations. The impact of identified mutations was assessed by sequence- and structure-based predictions and/or functional assays.

Results: We identified eight probands with $\mathrm{CHH}$ with $(n=3$; Kallmann syndrome) or without anosmia $(n=5)$ and SHFM, seven of whom (88\%) harbor FGFR1 mutations. Of these seven, one individual is homozygous for p.V429E and six individuals are heterozygous for p.G348R, p.G485R, p.Q594*, p.E670A, p.V688L, or p.L712P. All mutations were predicted by in silico analysis to cause loss of function. Probands with FGFR1 mutations have severe gonadotropinreleasing hormone deficiency (absent puberty and/or cryptorchidism and/or micropenis). SHFM in both hands and feet was observed only in the patient with the homozygous p.V429E mutation; V429 maps to the fibroblast growth factor receptor substrate $2 \alpha$ binding domain of FGFR1, and functional studies of the p.V429E mutation demonstrated that it decreased recruitment and phosphorylation of fibroblast growth factor receptor substrate $2 \alpha$ to FGFR1, thereby resulting in reduced mitogen-activated protein kinase signaling.

Conclusion: FGFR1 should be prioritized for genetic testing in patients with $\mathrm{CHH}$ and SHFM because the likelihood of a mutation increases from $10 \%$ in the general $\mathrm{CHH}$ population to $88 \%$ in these patients.

Genet Med advance online publication 13 November 2014

Key Words: congenital hypogonadotropic hypogonadism; fibroblast growth factor receptor 1; FGF receptor substrate $2 \alpha$; split hand/ foot malformation

\section{INTRODUCTION}

Congenital hypogonadotropic hypogonadism (CHH (OMIM 146110)) is a genetic disorder characterized by absent or incomplete pubertal development and infertility due to deficiency of gonadotropin-releasing hormone $(\mathrm{GnRH})$ secretion or action.
The co-occurrence of $\mathrm{CHH}$ with anosmia is termed Kallmann syndrome (KS (OMIM 308700, 147950, 244200, 610628, 612370 , and 612702)). Anosmia in KS is usually linked to agenesis of the olfactory structures, which provide the anatomic path for the migration of GnRH neurons from the olfactory

\footnotetext{
The first two authors contributed equally to this work.

${ }^{1}$ Unité Mixte de Recherche 1141, Institut National de la Santé et de la Recherche Médicale, Paris, France; ²Université Paris Diderot, Sorbonne Paris Cité, Hôpital Robert Debré, Paris, France; ${ }^{3}$ Harvard Reproductive Endocrine Sciences Center and the Reproductive Endocrine Unit of the Department of Medicine, Massachusetts General Hospital, Boston, Massachusetts, USA; ${ }^{4}$ Department of Pediatrics, Division of Pediatric Endocrinology, State University of New York Downstate Medical Center, Brooklyn, New York, USA; ${ }^{5}$ Department of Endocrinology and Diabetes, Shanghai Huashan Hospital, Shanghai, China; ${ }^{6}$ Department of Endocrinology, Diabetology and Metabolism, Lausanne University Hospital (CHUV), Lausanne, Switzerland; ${ }^{7}$ Faculty of Biology and Medicine, University of Lausanne, Lausanne, Switzerland; ${ }^{8}$ Service de Génétique Clinique, Hôpital Jeanne de Flandre, Lille, France; ${ }^{9}$ Department of Biochemistry and Molecular Pharmacology, New York University School of Medicine, New York, New York, USA; ${ }^{10}$ Institute of Biomedicine/ Physiology, University of Helsinki and Children's Hospital, Helsinki University Central Hospital, Helsinki, Finland; ${ }^{11}$ Centre for Neuroendocrinology, University College London Medical School, London, UK; ${ }^{12}$ Division of Pediatric Endocrinology, Meyer Children's Hospital, Rambam Health Care Campus, Haifa, Israel; ${ }^{13}$ Bruce Rappaport Faculty of Medicine, Institute of Technology, Haifa, Israel; ${ }^{14}$ Clinical Genetics, Hôpital Universitaire de la Côte de Nacre, Caen, France; ${ }^{15}$ Service d'Endocrinologie Diabétologie Pédiatrique et Centre de Référence des Maladies Endocriniennes Rares de la Croissance, Assistance Publique-Hôpitaux de Paris, Hôpital Robert Debré, Paris, France; ${ }^{16}$ Department of Genetics, Rouen University Hospital, Rouen, France; ${ }^{17}$ Department of Endocrinology, University Hospital of Rouen, Institute for Biomedical Research, University of Rouen, Rouen, France; ${ }^{18}$ Service d’Endocrinologie Gynécologie Diabétologie Pédiatriques, Hôpital Universitaire Necker Enfants Malades, University Paris Descartes, Paris, France; ${ }^{19}$ Endocrinology-Diabetology Unit, Department of Pediatrics, Lausanne University Hospital (CHUV), Lausanne, Switzerland; ${ }^{20}$ Laboratoire de Biochimie, Assistance Publique-Hôpitaux de Paris, Hôpital Robert Debré, Paris, France. Correspondence: Nicolas de Roux (Nicolas.deroux@inserm.fr) or Nelly Pitteloud (Nelly.Pitteloud@chuv.ch)
} 
placode to the hypothalamic region during embryonic development. ${ }^{1}$ To date, mutations in $>20$ genes have been found to underlie $\mathrm{CHH}$, acting either alone or in combination. ${ }^{2}$

Approximately $10-12 \%$ of patients with $\mathrm{CHH}$ carry lossof-function mutations in fibroblast growth factor receptor 1 (FGFR1), the first gene reported to be associated with both KS and normosmic $\mathrm{CHH} .{ }^{3,4} \mathrm{CHH}$-associated FGFR1 mutations are typically heterozygous, and the disease is inherited as an autosomal dominant trait with variable expressivity. FGFR1 encodes a member of the FGFR subfamily of receptor tyrosine kinases. Upon binding a fibroblast growth factor ligand in the presence of heparan sulfate, FGFR1 dimerizes and its kinase domains are autophosphorylated. In turn, this activates intracellular pathways that culminate in diverse biological responses; activation of the phospholipase $\mathrm{C} \gamma$ pathway requires phosphorylation of FGFR1 tyrosine 766, whereas activation of the Ras-mitogenactivated protein kinase (MAPK) and phosphoinositide 3-kinase pathways is mediated by recruitment of FGF receptor substrate $2 \alpha$ (FRS2 $\alpha){ }^{5}$ FGFR1 is expressed in multiple tissues, including the brain and skeleton; ${ }^{6}$ among other functions, it is required for fate specification of $\mathrm{GnRH}$ neurons in the olfactory placode, as well as for GnRH neuron proliferation and migration to the hypothalamus. ${ }^{7}$ Alternative splicing of extracellular region-encoding exons of FGFR1 gives rise to the FGFR1b and FGFR1c isoforms; to date, the majority of $\mathrm{CHH}$-associated mutations implicate FGFR1c as the major isoform relevant to GnRH neuron biology.,4

$\mathrm{CHH}$ patients with loss-of-function FGFR1 mutations are enriched for additional skeletal phenotypes, such as cleft lip/ palate, dental agenesis, mandibular hypoplasia, scoliosis, butterfly vertebrae, syndactyly, oligodactyly, and clinodactyly.,9 FGFR1 mutations (predicted to cause loss of function) have recently been identified in patients with Hartsfield syndrome (OMIM 615465), ${ }^{10}$ a rare disorder characterized by the association of holoprosencephaly, and split hand/foot malformation (SHFM; also called ectrodactyly), a severe malformation of skeletal development with an absent or incomplete development of the central rays of the hands, feet, or both. ${ }^{11}$ Notably, associated phenotypes, including midline defect, multiple pituitary hormone deficiency, and/or agenesis of the olfactory bulbs/tracts, have been described in patients with Hartsfield syndrome. ${ }^{10,12}$ Here we report the association of CHH with SHFM and show that the large majority of patients with both SHFM and $\mathrm{CHH}$ carry loss-of-function FGFR1 mutations.

\section{MATERIALS AND METHODS}

\section{Patients}

Via international collaboration (France, the United Kingdom, Finland, and the United States), we identified eight patients with $\mathrm{CHH}$ and SHFM (seven males and one female). Diagnostic criteria for $\mathrm{CHH}$ included (i) failure to initiate and/or complete spontaneous puberty by age 18 years; (ii) serum testosterone $\leq 3 \mathrm{nmol} / \mathrm{l}$ for men or estradiol $\leq 0.07 \mathrm{nmol} / \mathrm{l}$ for women, with low or normal serum concentrations of gonadotropins; (iii) otherwise normal pituitary function (absence of clinical and/or biochemical evidence of thyroid-stimulating hormone, adrenocorticotropic hormone, or growth hormone deficiency, hyperprolactinemia, or diabetes insipidus), and (iv) normal magnetic resonance imaging of the hypothalamic-pituitary region; or, in infants, (v) micropenis and/or cryptorchidism in the setting of low sex steroid and gonadotropin concentrations during "minipuberty."13 Spontaneous partial pubertal development was assessed based on clinical history, Tanner stage, and (in males) testicular size. Olfaction was assessed by self-report and/ or formal smell testing (brief smell identification test or olfactometry). Skeletal phenotypes assessed included SHFM, cleft lip/palate, and dental agenesis. The institutional review boards/ ethics committees of the Massachusetts General Hospital, Hôpital Robert Debré, Helsinki University Central Hospital, and University College London Medical School approved the studies; all subjects or parents/legal guardians provided written informed consent.

\section{Sequencing}

Genomic DNA was obtained from peripheral blood samples using standard phenol-chloroform extraction. Mutation screening for FGFR1 (NM_023110.2) was performed as previously described. ${ }^{2}$ The coding exonic and proximal intronic $(\geq 15$ base pairs from splice sites) DNA sequences of FGFR1 were amplified by polymerase chain reaction and analyzed by direct sequencing. Sequence variations were found on both DNA strands and were confirmed by a separate polymerase chain reaction. Variants were considered pathogenic mutations if (i) their allele frequency was $<1 \%$ in the 1000 Genomes Project data set and in the Exome Variant Server, and (ii) the altered amino acid was predicted to cause loss of function by structural modeling ${ }^{14}$ or by at least two prediction programs: PolyPhen-2 (ref. 15), SIFT, ${ }^{16}$ PMut, ${ }^{17}$ Mutation Taster ${ }^{18}$ and Condel ${ }^{19}$ for missense variants. All subjects were also screened for the presence of mutations in FGF8 (OMIM 600483). Other CHH genes were selectively sequenced in some subjects (Supplementary Data online): KAL1 (OMIM 300836), PROKR2 (OMIM 607123), PROK2 (OMIM 607002), TACR3 (OMIM 162332), TAC3 (OMIM 162330), GNRHR (OMIM 138850), GNRH1 (OMIM 152760), KISS1R (OMIM 604161), KISS1 (OMIM 603286), NSMF (OMIM 60813), CHD7 (OMIM 608892), and HS6ST1 (OMIM 604846) (primers and polymerase chain reaction conditions are available upon request).

\section{Structural modeling}

To predict the functional consequences of the identified FGFR1 mutations, the following structures were used: (i) crystal structure of the extracellular ligand-binding domain of human FGFR1 in complex with human FGF2 (Protein Data Bank identifier 1FQ9 ${ }^{20}$; (ii) crystal structure of the phosphorylated tyrosine kinase domain of human FGFR1 (Protein Data Bank identifier $3 \mathrm{GQI})^{21}$; and (iii) nuclear magnetic resonance solution structure of a 22 residue-long peptide derived from the juxtamembrane region of FGFR1 (residues 409-430) in complex with the FRS2 $\alpha$ phosphotyrosine binding (PTB) domain (Protein Data Bank 
identifier 1XR0).22 The structures were analyzed using Program $\mathrm{O}$, and structural representations were prepared using PyMol.

\section{Analysis of recruitment and phosphorylation of FRS2 $\alpha$ by FGFR}

Cell-based FRS2 phosphorylation assay. We first evaluated the effect of the V429E mutation on the ability of FGFR1c to phosphorylate FRS2 using a cell-based assay. Wild type (WT) and V429E FGFR1c were cloned into the lentiviral vector FUCRW following standard protocols. BaF3 cells were maintained as described previously $y^{23}$ and were transfected with FGFR1 pseudo-viral stock in Hanks balanced salt solution buffer. Stably transduced cells were treated with $1.5 \mathrm{nmol} / \mathrm{l}$ of FGF1 for $10 \mathrm{~min}$, rinsed in phosphate-buffered saline, and then lysed in radioimmunoprecipitation assay buffer (Thermo Scientific, Cambridge, UK). Cell extract $(30 \mu \mathrm{g})$ was resolved by sodium dodecyl sulfate-polyacrylamide gel electrophoresis and analyzed by western blotting using anti-FGFR1 (in-house antibody raised in rabbit), anti-FRS2 (ab10425; Abcam), and anti-phospho-FRS2- $\alpha$ (Tyr196) (no. 3864; Cell Signaling Technology, Danvers, MA) antibodies.

Surface plasmon resonance assay. FGFR2 and FRS2 peptides were prepared and surface plasmon resonance spectroscopy was analyzed following published protocols (for details, see the Supplementary Materials online). ${ }^{24}$

In vitro FRS2 phosphorylation assay. To study the impact of the V430E mutation on the phosphorylation of FRS2 $\alpha$, FGFR2CD ${ }^{\mathrm{WT}}(2 \mu \mathrm{mol} / \mathrm{l})$ or FGFR2CD ${ }^{\mathrm{V} 430 \mathrm{E}}$ was mixed with FRS $2 \alpha^{\text {PTB }}(40 \mu \mathrm{mol} / \mathrm{l})$ in a reaction buffer consisting of 25 $\mathrm{mmol} / \mathrm{l}$ adenosine triphosphate, $50 \mathrm{mmol} / \mathrm{l}$ magnesium chloride, $25 \mathrm{mmol} / \mathrm{l}$ HEPES (pH 7.5), and $150 \mathrm{mmol} / \mathrm{l}$ sodium chloride at ambient temperature. Reactions were quenched at different time points by the chelating the $\mathrm{Mg}^{2+}$ with equal moles of ethylenediaminetetraacetic acid. Following tryptic digestion, the amount of a phospho-Y196-containing peptide derived from FRS $2 \alpha^{\text {PTB }}$ was quantified by Orbitrap mass spectrometry and expressed as a fraction of the total amount of Y196containing tryptic peptide.

FGFR1 signaling reporter gene assays. The activation of downstream signaling pathways by WT and mutated FGFR1 constructs was interrogated using two firefly luciferase-based reporter bioassays: the osteocalcin FGF response element reporter, which reports activity of the MAPK pathway downstream of FRS2 $\alpha$ signaling, ${ }^{14}$ and a nuclear factor of activated T cells reporter (plasmid 10959; Addgene, Cambridge, MA), ${ }^{25}$ which reports activity of the phospholipase $\mathrm{C} \gamma /$ inositol trisphosphate/ $\mathrm{Ca}^{2+}$ cascade independent of FRS $2 \alpha .{ }^{26}$ Transient transfection experiments in L6 myoblasts and luciferase assays were performed as previously described. ${ }^{14}$ Each experiment was performed in triplicate and repeated three or more times. The data were fitted with three-parameter sigmoidal curves using Prism5 (GraphPad Software, San Diego, CA) and the dose-response curves of mutant receptors were compared with those of WT FGFR1 using the Prism5 F-test function.

FGFR1 protein abundance, maturation, and cell surface expression assays. The FGFR1 V429E mutation was subcloned into the previously described FGFR1c expression construct myc-FGFR $1^{\text {WT }}$, which incorporates an $\mathrm{N}$-terminal myc tag for antibody-mediated detection. ${ }^{14}$ The impacts of the mutation on the total abundance, folding, and cell surface expression of the receptor were assayed in COS-7 cells as previously described; ${ }^{14}$ results of three experiments, each performed in quadruplicate, were compared using the Mann-Whitney $U$-test.

\section{RESULTS}

\section{Loss-of-function FGFR1 mutations are highly prevalent in patients with $\mathrm{CHH}$ and SHFM}

We identified eight $\mathrm{CHH}$ probands (seven males and one female) with SHFM, including three patients with KS (Table 1, Supplementary Data online). Seven mutations in FGFR1 were identified in seven male $\mathrm{CHH}$ probands (Table 1; Figure 1); thus $88 \%$ of patients with $\mathrm{CHH}$ and SHFM harbor mutations in FGFR1. Among the probands with FGFR1 mutations, four also exhibit cleft palate; five cases are clearly familial (families 1-4 and 7; Figure 1). The proband exhibiting the most severe SHFM phenotype (both hands and feet affected, with additional syndactylies) carries the homozygous missense mutation p.V429E. ${ }^{27}$ By contrast, six probands with SHFM (limited to either one foot or both feet) carry heterozygous mutations, five missense and one nonsense: p.G348R, p.G485R, p.Q594*, p.E670A, p.V688L, and p.L712P (Table 1). Two mutations (p.G348R and p.E670A) have been previously reported ${ }^{28,29}$

Previously reported mutations identified in patients with $\mathrm{CHH}$ are distributed evenly between the extracellular and intracellular domains (Figure 2a). By contrast, the majority of the FGFR1 mutations (five of seven) in the probands with $\mathrm{CHH}$ and SHFM affect amino acids located in the tyrosine kinase domain (Figure 2b). The nonsense mutation (p.Q594*) is expected to lead to synthesis of a truncated inactive receptor that lacks the part of the tyrosine kinase domain containing the catalytic site. For missense mutations, the affected residues are conserved across vertebrates (Figure $\mathbf{2 b}$ ), and all mutations are predicted to cause loss of function (Supplementary Table S1 online; Supplementary Figure S1 online, Supplementary Data online). p.E670A impairs FGFR1 downstream signaling as assessed by an in vitro MAPK phosphorylation assay. ${ }^{29}$ p.V429E is the first reported mutation within the domain of FGFR1 that binds FRS2 $\alpha$, the docking protein that mediates MAPK pathway activation ${ }^{30}$ (Figure 3a, Supplementary Data online).

\section{FGFR1-V429E substitution abolishes recruitment and phosphorylation of FRS2 $\alpha$ by the FGFR}

We chose to functionally verify our structural predictions on the p.V429E substitution because this is the first report of a mutation that would selectively affect a specific signaling pathway 


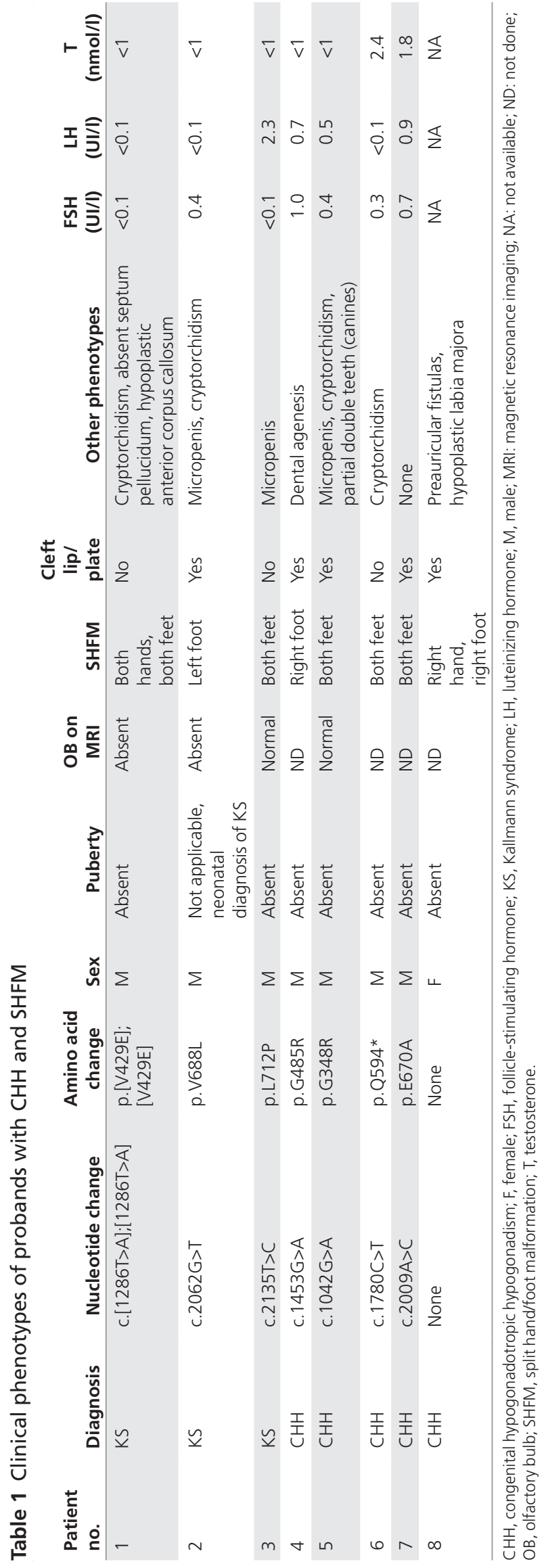

downstream of FGFR. A lentiviral expression system was used to express the WT FGFR1c and its V429E variant in BaF3 cells, which lack endogenous FGFR expression. Cells were transfected with the WT FGFR1c or the V429E FGFR1c mutant constructs and treated with FGF1; FRS2 $\alpha$ phosphorylation on tyrosine 196, one of the major Grb2 binding site of FRS2 $\alpha$, was assessed by western blotting with phosphospecific antibodies. As shown in Figure 3b, WT FGFR1c phosphorylated FRS2 $\alpha$ on tyrosine 196, whereas the V429E mutant did not. For in vitro binding and phosphorylation experiments, we used the FGFR2 intracellular domain. This was necessary because the expression level of the FGFR1 intracellular domain in Escherichia coli is too low to allow these experiments to be performed. By contrast, the FGFR2 intracellular domain can be expressed abundantly and can be purified to high homogeneity using an E. coli expression system. This approach is legitimate because FGFR1 and FGFR2 are structurally and functionally highly homologous. The FRS2 $\alpha$ binding sites are highly conserved between these FGFRs; alternative splicing in both FGFRs, and the exclusion of the conserved Val-Thr motif, eliminates FRS2 $\alpha$ recruitment to the FGFR. ${ }^{31}$ WT FGFR2 intracellular domain (residues 401-821; FGFR2CD ${ }^{\mathrm{WT}}$ ), the corresponding mutated fragment (FGFR2CD ${ }^{\mathrm{V} 430 \mathrm{E}}$ ), and the PTB domain of FRS2 $\alpha$ (residues 11140; FRS2 $\alpha^{\mathrm{PTB}}$ ) were expressed in E. coli as His-tagged proteins and purified to homogeneity. FRS2 $\alpha$ was immobilized on a CM5 chip sensor, and increasing concentrations of FGFR2CD ${ }^{\mathrm{WT}}$ or FGFR2CD ${ }^{\mathrm{V} 30 \mathrm{E}}$ were flowed over the sensor chip. FGFR2CD ${ }^{\mathrm{WT}}$ bound FRS $2 \alpha$ P with a dissociation constant $\left(K_{\mathrm{D}}\right)$ of $320 \mathrm{nmol} / \mathrm{l}$, whereas the FGFR2CD ${ }^{\mathrm{V} 430 \mathrm{E}}$ mutant failed to bind to FRS2 $\alpha^{\mathrm{PTB}}$ (Figure 3c). Using an in vitro kinase assay, we also examined the effect of the mutation on the ability of the mutant receptor to phosphorylate FRS2 $\alpha$. Briefly, purified FRS2 $\alpha$ protein was mixed with FGFR2CD ${ }^{\mathrm{WT}}$ or FGFR2CD ${ }^{\mathrm{V} 430 \mathrm{E}}$ in a molar ratio of 20:1 and supplemented with a magnesium-adenosine triphosphate mixture to start the phosphorylation reaction. Phosphorylation of FRS2 $\alpha$ on tyrosine 196 as a function of time was monitored by mass spectrometry and was expressed as percentage of the phosphorylated substrate. As shown in Figure 3d, the V430E mutation diminishes the ability of FGFR2 to phosphorylate FRS2 $\alpha$. These data demonstrate that the FGFR2V430E substitution diminishes the ability of FGFR2 to recruit and phosphorylate FRS2 $\alpha$ in vitro. In contrast to cell-based data, the mutation did not abolish FRS2 $\alpha$ phosphorylation in vitro. This was expected because the high concentrations of FGFR kinase and FRS2 $\alpha$ (several orders of magnitude greater than those in cell-based experiments) have resulted in recruitmentindependent phosphorylation of FRS2 $\alpha$ by the FGFR kinase. Collectively, these cell-based and in vitro data demonstrate that the FGFR1-V429E substitution diminishes the ability of FGFR1 to recruit and phosphorylate FRS2 $\alpha$.

\section{FGFR1 V429E substitution compromises FGFR1 MAPK signaling in vitro}

We then studied the effects of the mutation on downstream signaling using the osteocalcin FGF response element and nuclear 
a

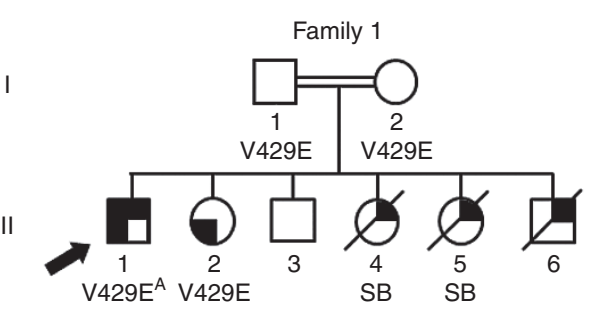

Family 3

I

II

III

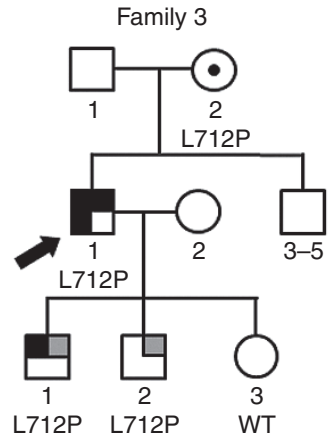

Family 2

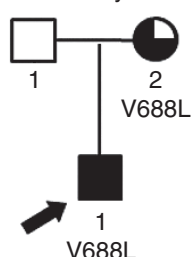

Family 4
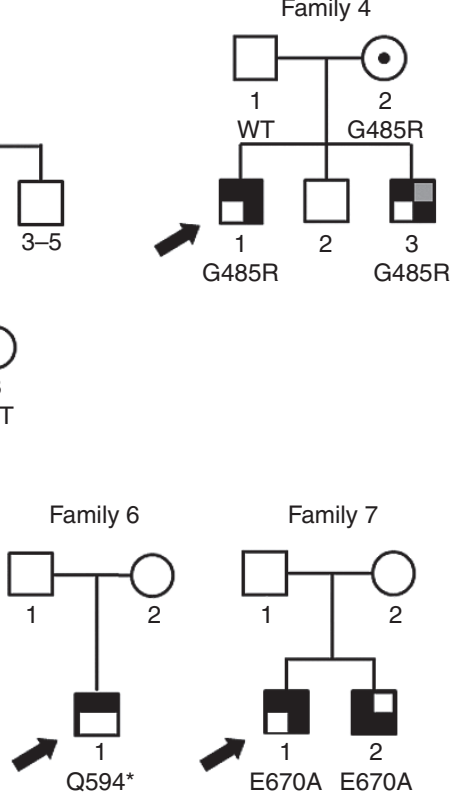

Family 7

Family 6

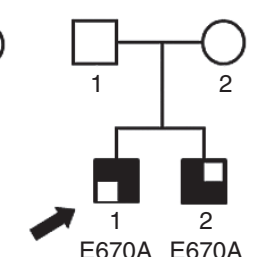

b

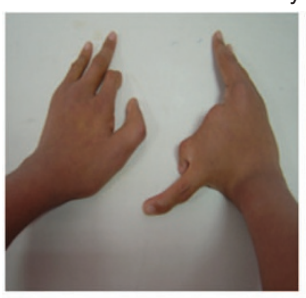

Family $1, \mathrm{II}-1$

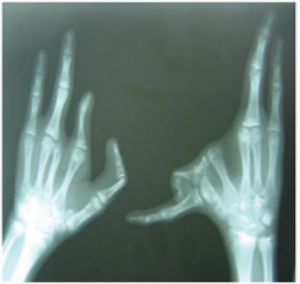

Family 5, II-1

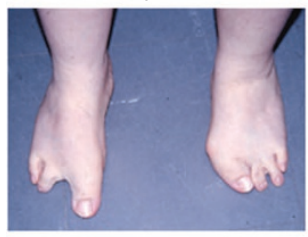

Family 5

।

II

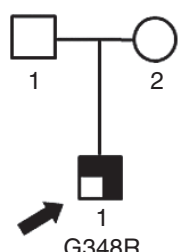

\begin{tabular}{|lll|}
\hline$\square \mathrm{CHH}$ & $\square$ SHFM & $\square$ Anosmia \\
$\square$ Cleft palate & $\square$ Syndactyly & $\bigcirc$ Asymptomatic carrier \\
\hline
\end{tabular}

Figure 1 FGFR1 mutations underlie congenital hypogonadotropic hypogonadism (CHH) with split hand/foot malformation (SHFM). (a) Pedigrees of the seven $\mathrm{CHH}$ and SHFM families with FGFR1 mutations; probands are denoted by arrows. (b) Photographs and radiographs demonstrating severe skeletal anomalies of hands and feet among probands. A, homozygous mutation; SB, stillborn.

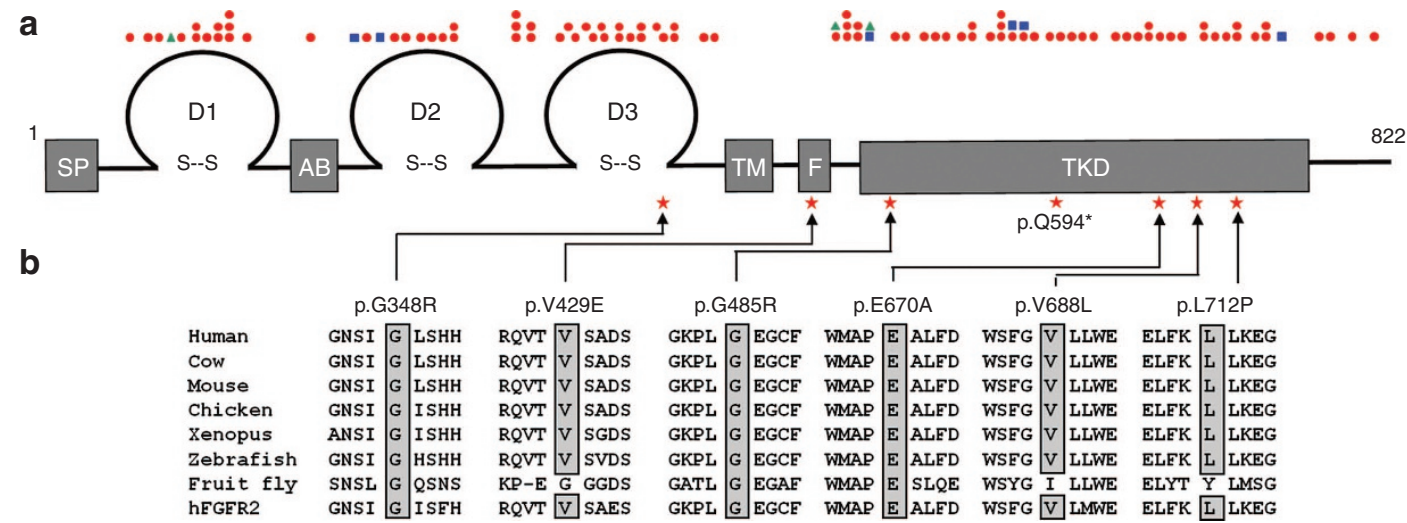

Figure 2 FGFR1 mutations identified in probands with congenital hypogonadotropic hypogonadism (CHH) and split hand/foot malformation (SHFM). (a) Schematic of FGFR1 showing the locations of all published FGFR1 mutations associated with CHH (Kallmann syndrome or normosmic CHH, red circles), Hartsfield syndrome (blue squares), septo-optic dysplasia (green triangles). (b) FGFR1 mutations identified in probands with CHH and SHFM; all the substituted residues of missense mutations are conserved across vertebrates (cow, mouse, chicken, Xenopus, and zebrafish) and in human FGFR2 (hFGFR2). $A B$, acid box domain; D1, immunoglobulin domain 1; D2, immunoglobulin domain 2; D3, immunoglobulin domain 3; F, FRS2 $\alpha$-binding domain; SP, signal peptide; stars, mutations described in this study; TKD, tyrosine kinase domain; TM, transmembrane domain. 
a

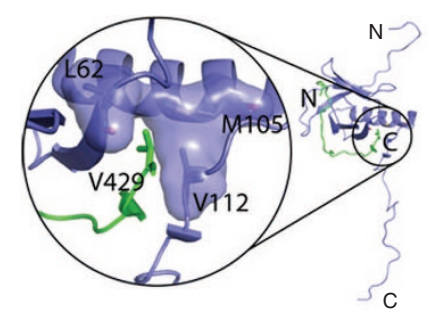

e

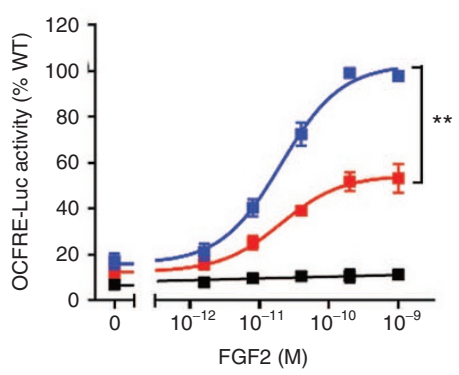

b

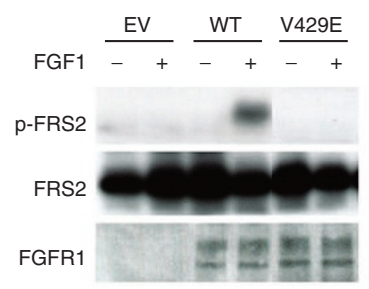

f

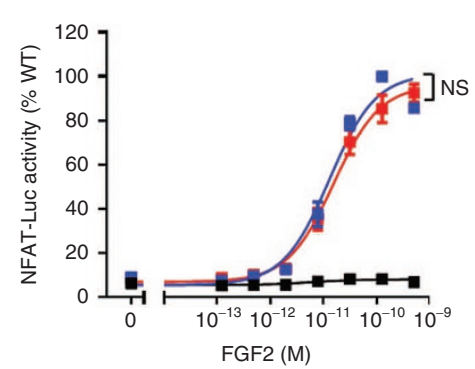

c

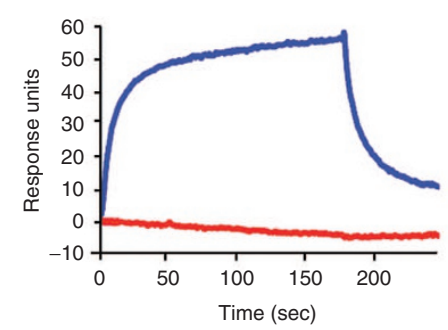

g

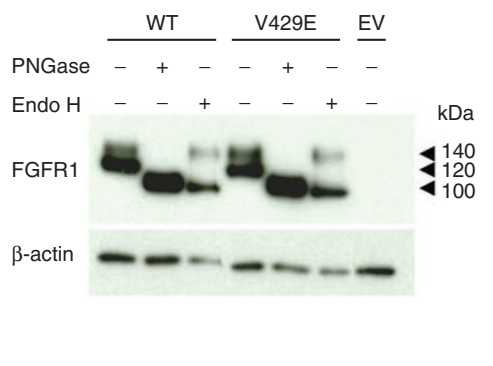

d

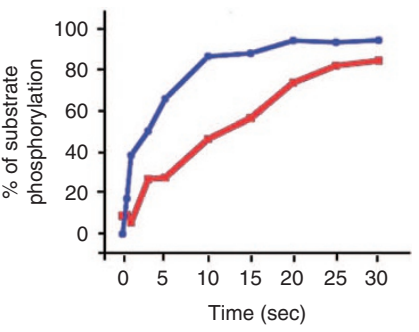

h

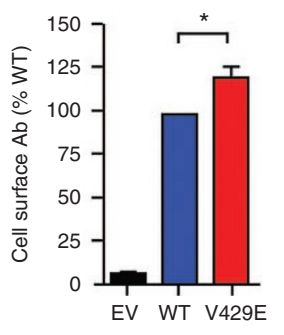

Figure 3 The V429E substitution in FGFR1 impedes recruitment and phosphorylation of FRS2 $\alpha$ and FGF2-induced mitogen-activated protein kinase signaling. (a) Analysis of the impact of the V429E mutation based on the nuclear magnetic resonance structure of the FRS2 phosphotyrosine binding (PTB) domain in complex with the juxtamembrane (JM) region peptide of FGFR1. The FRS2 PTB domain and FGFR1 JM peptide are shown as purple and green ribbons, respectively, and side chains of the V429 of FGFR1 and L62, M105, and V112 of FRS2 are rendered as sticks. The molecular surfaces of L62, M105, and V112 of FRS2 PTB are also shown to highlight their hydrophobic contacts with V429 of FGFR1. (b) The V429E FGFR1c mutant fails to phosphorylate FRS2 in cellbased assay. BaF3 cells were transfected with lentiviral vectors expressing wild-type (WT) or V429E FGFR1C, and FRS2 phosphorylation upon FGF1 treatment was assessed by western blotting using anti-phospho-FRS2- $\alpha$-specific antibodies. EV, empty vector. (c) Analysis of the impact of the V429E mutation on the ability of FGFR1 to recruit FRS2 $\alpha$. The assay was based on FGFR2 V430E, which is equivalent to FGFR1 V429E. Increasing concentrations of FGFR2CDWT and FGFR2CDV430E (carrying the equivalent mutation to FGFR1-V429E), ranging from 12.5 to $400 \mathrm{nmol} / \mathrm{l}$, were passed over a CM5 chip upon which FRS2 $\alpha$ had been immobilized. As representative of the full data set, binding responses obtained for injections of $200 \mathrm{nmol} / \mathrm{l}$ of FGFR2CDWT or FGFR2CD ${ }^{\mathrm{V} 30 \mathrm{E}}$ are shown. The rising and falling parts of the WT curve (blue) represent the association and dissociation phases, respectively, of FGFR2CDWT_FRS2 $\alpha$ binding over time. At 200 nmol/l, FGFR2CDWT exhibits maximal binding of 55 response units (blue), whereas FGFR2CDV430E shows negligible binding (red). According to a steady-state equilibrium analysis of the full data sets (not shown), FGFR2CDWT binds FRS2 $\alpha$ with a dissociation constant $\left(K_{D}\right)$ of $320 \mathrm{nmol} / \mathrm{l}$, whereas the FGFR2CDV430E mutant has negligible binding to FRS2 $\alpha$. (d) The V429E mutation reduces the ability of FGFR1 to phosphorylate FRS2 on Y196 in vitro. FGFR2 ${ }^{W T}$ and FGFR2 ${ }^{\mathrm{V} 30 \mathrm{E}}$ mutant kinases were allowed to phosphorylate FRS2 fragment PTB ${ }^{9-200}$ on Y196, a tyrosine phosphorylation site known to be required for Grb2 recruitment, at room temperature for 0, 1, 3, 5 10, $15,20,25$, or 30 minutes. Following tryptic digestion, samples were analyzed by Orbitrap mass spectrometry to quantify the phospho-Y196-containing tryptic peptide. FGFR2 ${ }^{\text {WT }}$ is shown in blue and FGFR2 ${ }^{\mathrm{V} 330 \mathrm{E}}$ is shown in red. (e,f) The V429E mutation caused loss of function in the osteocalcin FGF response element reporter assay (FRS2 $\alpha$-dependent mitogen-activated protein kinase signaling) and is not different from WT in the nuclear factor of activated T-cells reporter assay (FRS2 $\alpha$-independent phospholipase $\mathrm{C} \gamma /$ inositol trisphosphate/C $\mathrm{C}^{2+}$ signaling). Data shown represent the means \pm SEM of three experiments. FGFR1 WT is shown in blue, FGFR1 ${ }^{\mathrm{V} 229 \mathrm{E}}$ in red, EV in black. Relative to the maximal stimulation of WT (percentage), $* \star P<0.001$. NS, not significant. (g) Total abundance and maturation of recombinant FGFR1 proteins. COS-7 cells were transfected with FGFR1 constructs and the cell lysates were subjected to deglycosylation treatment followed by western blot analysis. Bands treated with peptide N-glycosidase F represent total protein abundance; $140-\mathrm{kDa}$ endoglycosidase $\mathrm{H}$ (Endo H)-treated bands represent the mature form, whereas $100-\mathrm{kDa}$ Endo $\mathrm{H}$-treated bands represent the immature form of FGFR1. The experiment was performed three times and tested with the Mann-Whitney U-test for statistic significance; no significant difference in overall expression and maturation index between WT and V429E was found. (h) Cell surface abundance of the transiently transfected FGFR1 mutant in COS-7 cells. Cell surface abundance was measured by a radiolabeled antibody binding assay and plotted as a percentage of WT levels. The abundance of FGFR1 ${ }^{\mathrm{V} 429 \mathrm{E}}$ expressed on the cell surface was significantly higher than that of FGFR1 ${ }^{\text {WT }}$. Values shown are the means \pm SEM of three experiments, each performed in quadruplicate. The difference between the mutant and the WT receptor expression was compared using the Mann-Whitney $U$-test, ${ }^{*} P<0.05$.

factor of activated $\mathrm{T}$ cells reporter systems. Stimulation of the WT receptor with increasing doses of FGF2 resulted in a typical sigmoidal dose-response curve with approximately fivefold maximal induction of reporter activity (Figure 3e). The V429E mutant displayed reduced inducibility: $56 \%$ of WT at maximum activity $(P<0.001$; Figure $3 e)$; this suggests compromised MAPK signaling. By contrast, V429E behaved similarly to the WT receptor in the nuclear factor of activated T-cells reporter assay (Figure 3f), indicating normal phospholipase $\mathrm{C} \gamma /$ inositol trisphosphate/ $\mathrm{Ca}^{2+}$ signaling. These findings indicate that V429E represents a partial loss-of-function mutation that compromises the activation of FRS2 $\alpha$-dependent MAPK signaling without affecting FRS2 $\alpha$-independent phospholipase $\mathrm{C} \gamma /$ inositol trisphosphate/ $\mathrm{Ca}^{2+}$ signaling by FGFR1.

To exclude the possibility that the V429E mutation leads to a loss of function by impairing FGFR1 receptor protein synthesis and/or maturation, we investigated whether it affected the total abundance, glycosylation, or cell surface expression of FGFR1. When the lysate of transiently transfected COS-7 cells was subjected to western blot analysis under reducing conditions, FGFR1 was detectable as two protein bands of 140 and $120 \mathrm{kDa}$ (Figure 3g). When the lysate was pretreated with 
peptide $\mathrm{N}$-glycosidase $\mathrm{F}$, which removes all N-linked carbohydrate chains, FGFR1 was detectable as a single $100-\mathrm{kDa}$ band, confirming that the two bands are differently N-glycosylated receptor pools. Total protein abundance was calculated as the ratios of peptide $\mathrm{N}$-glycosidase $\mathrm{F}$-treated bands to $\beta$-actin band densities; the value calculated for the V429E mutant was normalized to the WT receptor. Treatment with endoglycosidase $\mathrm{H}$, which removes only high-mannose $\mathrm{N}$-linked sugars, altered the mobility of only the 120-kDa FGFR1 band (Figure 3g), indicating that this pool represents the partially processed immature receptor, whereas the endoglycosidase $\mathrm{H}$-resistant $140-\mathrm{kDa}$ band represents the mature form of FGFR1. Receptor "maturation index" was estimated by calculating the ratio of the $140-\mathrm{kDa}$ band to the total FGFR1 immunoreactivity of endoglycosidase $\mathrm{H}$-treated samples. The total abundance (mean \pm SEM, $1.07 \pm 0.30 ; P>0.05$ ) and the maturation index $(1.04 \pm 0.05 ; P>0.05)$ of $\mathrm{V} 429 \mathrm{E}$ were not different from those of the WT, suggesting that the mutation did not compromise receptor synthesis or maturation. The cell surface abundance of V429E was slightly elevated (by $20 \%$ relative to WT; $P<0.05$ ) (Figure $3 \mathrm{~h}$ ), thus excluding reduced expression as a cause of decreased signaling activity. Hence, the V429E mutation imparted loss of receptor function by selectively inhibiting the ability of the receptor to interact with its major downstream substrate FRS2 $\alpha$ and to transduce respective intracellular signals.

\section{Variable expressivity of phenotypes associated with FGFR1 mutations}

In family 1, the proband with the homozygous mutation (p.V429E) exhibits severe reproductive, olfactory, and skeletal phenotypes (SHFM in the hands and feet and syndactylies). The only detectable phenotype among the three heterozygous family members analyzed is hyposmia in the proband's sister. In family 2, the proband with KS, SHFM, and cleft lip and palate inherited the heterozygous mutation (p.V688L) from his affected mother, who also exhibits KS and cleft lip and palate but not SHFM. In family 3, the proband with KS and SHFM inherited the heterozygous mutation (p.L712P) from his unaffected mother; his two sons (III-1 and III-2), who carry the mutation, both have syndactyly and one has $\mathrm{CHH}$. In family 4, the proband with $\mathrm{CHH}, \mathrm{SHFM}$, and cleft palate inherited his heterozygous mutation (p.G485R) from his unaffected mother. His brother, who carries the same mutation, has $\mathrm{CHH}$ and was born with syndactyly and cleft palate. Finally, in family 7, the proband who carries the p.E670A mutation has $\mathrm{CHH}$, SHFM, and cleft palate. DNA from his parents was not available. His affected brother, who has the identical FGFR1 mutation, has KS and cleft palate. Thus, among the five familial cases, SHFM was found in the probands with $\mathrm{CHH}$ and in three additional members in family 1 whose genotype and reproductive phenotype are unknown (two stillborn females and one male who died as a neonate). On the other hand, $\mathrm{CHH}$, anosmia/hyposmia, syndactyly, and cleft lip/palate occurred in several family members carrying the FGFR1 mutation (Figure 1).

\section{DISCUSSION}

We show here that FGFR1 mutations are present in most cases of CHH with SHFM (88\%). Several patients also exhibited anosmia (patients 1,2, and 3), cleft palate (patients 2, 4, 5, and 7), or, more rarely, absent septum pellucidum and hypoplastic anterior corpus callosum (patient 1), phenotypes previously reported in association with FGFR1 mutations., ${ }^{3,4}$ FGFR1 mutations have been recently reported to underlie Hartsfield syndrome, defined as the combination of holoprosencephaly and SHFM. ${ }^{10}$ A broad radiologic spectrum has been reported in holoprosencephaly, including absence of septum pellucidum and corpus callosum at the milder end. ${ }^{32}$ This phenotype was observed in patient 1 with $\mathrm{CHH}$ and SHFM. Furthermore, patients with Hartsfield syndrome may have olfactory bulb agenesis and/or multiple pituitary hormone deficiency (including hypogonadotropic hypogonadism). ${ }^{10,12}$ Thus, our results show a substantial phenotypic and genetic overlap between $\mathrm{CHH}$ with SHFM on the one hand and Hartsfield syndrome on the other. Indeed, FGFR1 is a pleiotropic gene not only involved in the development of the GnRH neuron ontogeny and the olfactory system but also implicated in forebrain development and embryonic limb morphogenesis. It is interesting to note that seven of eight probands reported here are males, and all seven carried loss-offunction mutations in FGFR1. The sole patient with $\mathrm{CHH}$ and SHFM without an FGFR1 mutation is a female with ectrodactyly of the right hand and foot and cleft palate. A similar male predominance has been observed in Hartsfield syndrome. ${ }^{10}$

In the consanguineous family 1 , the disease seems to be inherited as a recessive trait, which is unusual for $\mathrm{CHH}$ associated with FGFR1 mutations. ${ }^{3}$ The proband carries a homozygous p.V429E mutation, whereas three family members are heterozygous for the mutation, and only one among them exhibits hyposmia, a phenotype that can be associated with $\mathrm{CHH}$. This may be because the p.V429E mutation causes only partial loss of function, such that two mutant alleles are needed to manifest the disease phenotype. In the other six families, FGFR1-associated CHH with SHFM was inherited as an autosomal dominant trait with incomplete penetrance. For example, among the informative pedigrees, the mutation was transmitted by unaffected mothers (families 3 and 4); additional mutation carriers without SHFM are present in families 2, 3, 4, and 7. However, although SHFM is classically described as an absence of the central ray, a broader range of limb/extremity abnormalities has been described within this entity, such as syndactyly. ${ }^{11}$ Accordingly, the apparent penetrance of FGFR1 mutations increases when the phenotype includes $\mathrm{CHH}$ and a limb abnormality-not just SHFM (e.g., two patients with $\mathrm{CHH}$ and one prepubertal individual in families 3 and 4 harbor FGFR1 mutations and exhibit syndactyly). Oligogenicity ${ }^{2,27}$ and/or interaction with environmental factors ${ }^{33}$ may contribute to the incomplete penetrance and variable expressivity of $\mathrm{CHH}$, SHFM with other limb extremity abnormalities, and olfactory phenotypes.

Several lines of evidence support the hypothesis that the SHFM in these CHH probands is the consequence of the FGFR1 
signaling defect. Fgfr1 restricts the number of cells in nascent limb buds and specifies digit placement and identity in developing limbs. ${ }^{34,35}$ Mice with inactivated Fgfrl in sonic hedgehogexpressing cells of developing limb buds lack the third digit in all forelimbs and hind limbs, which corresponds to the human SHFM phenotype. ${ }^{35}$ In addition, mice with inactivation of $\mathrm{Fgfr} 1$ in limb mesenchyme immediately after limb bud initiation exhibit fused/missing first and second digits, similar to the phenotype of patients 1,5 and 6 (ref. 34). These data are consistent with a critical role for FGFR1 in limb development and SHFM pathogenesis. To date, the only gene associated with isolated (nonsyndromic) SHFM in both mice and humans is TP63. ${ }^{36}$ Interestingly, the limb buds of $T p 63^{-/-}$mice have markedly decreased expression of $F g f 8$ in the apical ectodermal ridge, ${ }^{37}$ Fgf8 is required for proliferation of the underlying mesenchymal cells. Moreover, mice with double conditional knockout of $F g f 8$ and $\mathrm{Fgf4}$ in apical ectodermal ridge cells exhibit aplasia of both proximal and distal limbs. ${ }^{38}$ Finally, a locus for SHFM maps to a region that includes FGF8 (chromosome 10q24-q25). ${ }^{39}$ FGF8 is a potent ligand for FGFR1, and both FGF8 and FGFR1 loss-offunction mutations underlie $\mathrm{CHH}$ through defects in olfactory bulb or GnRH neuron development. ${ }^{40}$ Mutations in FGF8 are a rare cause of $\mathrm{CHH}(\sim 1 \%)$, and none were found in the eight $\mathrm{CHH}-\mathrm{SHFM}$ probands reported here.

The most severe limb extremity phenotype, with median clefts in both hands and both feet as well as multiple syndactylies, was observed in the patient with a homozygous FGFR1 mutation (p.V429E). The p.V429E mutation is the first one identified in the binding domain for FRS2 $\alpha$; structural and functional studies show that the mutation selectively abolishes the interaction of the receptor with FRS2 $\alpha$, an adapter critical for activation of the MAPK and phosphoinositide 3-kinase cascades. ${ }^{5}$ MAPK activation by FGF signaling is known to promote survival and neurite outgrowth in GnRH neurons. ${ }^{7}$ The p.V429E mutation suggests a role for FRS2 $\alpha$-mediated FGFR1 signaling, not only for GnRH neuron ontogeny but also for distal limb development in humans. The remaining six patients with heterozygous FGFR1 mutations exhibit milder SHFM phenotypes with a median cleft in either one foot $(n=2)$ or both feet $(n=4)$.

In conclusion, the association of CHH with SHFM is a clinical entity with a high frequency of FGFR1 mutations. A main limitation of this study is that it was not designed to assess the percentage of the general $\mathrm{CHH}$ population that presents SHFM; rather, the cases were assembled based on phenotypes that came to the attention of the many collaborating physicians. To address the exact prevalence of CHH with SHFM, systematic phenotyping of defined $\mathrm{CHH}$ cohorts is necessary, which is beyond the scope of this study. Nevertheless, our findings have implications for clinic practice. First, the high frequency of FGFR 1 mutations in patients with $\mathrm{CHH}$ and SHFM as compared with the general $\mathrm{CHH}$ population suggests that FGFR1 should be prioritized for genetic screening in patients with $\mathrm{CHH}$ and SHFM. Second, because diagnosis of $\mathrm{CHH}$ during infancy can facilitate early treatment with gonadotropins/GnRH to promote both gonadal development and future fertility in adulthood, ${ }^{13}$ we propose that neonates with SHFM be assessed for $\mathrm{CHH}$ through evaluation at birth for micropenis and cryptorchidism in males, hormonal testing during "minipuberty", magnetic resonance imaging of olfactory structures, and/or genetic screening of FGFR1. Finally, further refining the clinical and genetic overlap among $\mathrm{CHH}$, SHFM, and Hartsfield syndrome is expected to facilitate a better understanding of their pathologic mechanisms and to elaborate a rational algorithm for genetic diagnosis.

\section{SUPPLEMENTARY MATERIAL}

Supplementary material is linked to the online version of the paper at http://www.nature.com/gim

\section{ACKNOWLEDGMENTS}

The authors are grateful to all the patients and families for their kind participation and to Kemal Topaloglu for clinical and genetic information. This work was supported by the Eunice Kennedy Shriver National Institute of Child Health and Human Development of the National Institutes of Health (R01HD056264 to N.P., R01DE013686 to M.M.), Swiss National Science Foundation grants (31003A, 135648 to N.P. and CRSII3141960 to N.P. and M.M.), Academy of Finland grants (to T.R. and J.T.), INSERM, the DHU PROTECT, and COST Action BM1105.

\section{DISCLOSURE}

The authors declare no conflict of interest.

\section{REFERENCES}

1. Tsai PS, Gill JC. Mechanisms of disease: insights into X-linked and autosomaldominant Kallmann syndrome. Nat Clin Pract Endocrinol Metab 2006;2:160171.

2. Miraoui H, Dwyer AA, Sykiotis GP, et al. Mutations in FGF17, IL17RD, DUSP6, SPRY4, and FLRT3 are identified in individuals with congenital hypogonadotropic hypogonadism. Am J Hum Genet 2013;92:725-743.

3. Dodé C, Levilliers J, Dupont JM, et al. Loss-of-function mutations in FGFR1 cause autosomal dominant Kallmann syndrome. Nat Genet 2003;33:463-465.

4. Pitteloud N, Acierno JS Jr, Meysing A, et al. Mutations in fibroblast growth factor receptor 1 cause both Kallmann syndrome and normosmic idiopathic hypogonadotropic hypogonadism. Proc Natl Acad Sci USA 2006;103:62816286.

5. Eswarakumar VP, Lax I, Schlessinger J. Cellular signaling by fibroblast growth factor receptors. Cytokine Growth Factor Rev 2005;16:139-149.

6. Deng CX, Wynshaw-Boris A, Shen MM, Daugherty C, Ornitz DM, Leder P. Murine FGFR-1 is required for early postimplantation growth and axial organization. Genes Dev 1994;8:3045-3057.

7. Tsai PS, Moenter SM, Postigo HR, et al. Targeted expression of a dominantnegative fibroblast growth factor (FGF) receptor in gonadotropin-releasing hormone $(\mathrm{GnRH})$ neurons reduces FGF responsiveness and the size of $\mathrm{GnRH}$ neuronal population. Mol Endocrinol 2005;19:225-236.

8. Costa-Barbosa FA, Balasubramanian R, Keefe KW, et al. Prioritizing genetic testing in patients with Kallmann syndrome using clinical phenotypes. J Clin Endocrinol Metab 2013;98:E943-E953.

9. Jarzabek K, Wolczynski S, Lesniewicz R, Plessis G, Kottler ML. Evidence that FGFR1 loss-of-function mutations may cause variable skeletal malformations in patients with Kallmann syndrome. Adv Med Sci 2012;57:314-321.

10. Simonis N, Migeotte I, Lambert N, et al. FGFR1 mutations cause Hartsfield syndrome, the unique association of holoprosencephaly and ectrodactyly. $J$ Med Genet 2013;50:585-592.

11. Duijf PH, van Bokhoven $\mathrm{H}$, Brunner HG. Pathogenesis of split-hand/split-foot malformation. Hum Mol Genet 2003;12 Spec No 1:R51-R60.

12. Vilain C, Mortier G, Van Vliet G, et al. Hartsfield holoprosencephaly-ectrodactyly syndrome in five male patients: further delineation and review. Am J Med Genet A 2009;149A:1476-1481. 
13. Bouvattier C, Maione L, Bouligand J, Dodé C, Guiochon-Mantel A, Young J. Neonatal gonadotropin therapy in male congenital hypogonadotropic hypogonadism. Nat Rev Endocrinol 2012;8:172-182.

14. Raivio T, Sidis Y, Plummer L, et al. Impaired fibroblast growth factor receptor 1 signaling as a cause of normosmic idiopathic hypogonadotropic hypogonadism. J Clin Endocrinol Metab 2009;94:4380-4390.

15. Adzhubei IA, Schmidt S, Peshkin L, et al. A method and server for predicting damaging missense mutations. Nat Methods 2010;7:248-249.

16. Kumar P, Henikoff S, Ng PC. Predicting the effects of coding non-synonymous variants on protein function using the SIFT algorithm. Nat Protoc 2009;4:10731081.

17. Ferrer-Costa C, Orozco M, de la Cruz X. Sequence-based prediction of pathological mutations. Proteins 2004;57:811-819.

18. Schwarz JM, Rödelsperger C, Schuelke M, Seelow D. MutationTaster evaluates disease-causing potential of sequence alterations. Nat Methods 2010;7:575-576.

19. González-Pérez A, López-Bigas N. Improving the assessment of the outcome of nonsynonymous SNVs with a consensus deleteriousness score, Condel. Am J Hum Genet 2011;88:440-449.

20. Schlessinger J, Plotnikov AN, Ibrahimi OA, et al. Crystal structure of a ternary FGF-FGFR-heparin complex reveals a dual role for heparin in FGFR binding and dimerization. Mol Cell 2000;6:743-750.

21. Bae JH, Lew ED, Yuzawa S, Tomé F, Lax I, Schlessinger J. The selectivity of receptor tyrosine kinase signaling is controlled by a secondary $\mathrm{SH} 2$ domain binding site. Cell 2009;138:514-524.

22. Dhalluin C, Yan KS, Plotnikova O, et al. Structural basis of SNT PTB domain interactions with distinct neurotrophic receptors. Mol Cell 2000;6:921-929.

23. Ornitz DM, Xu J, Colvin JS, et al. Receptor specificity of the fibroblast growth factor family. J Biol Chem 1996;271:15292-15297.

24. Ibrahimi OA, Zhang F, Eliseenkova AV, Itoh N, Linhardt RJ, Mohammadi M. Biochemical analysis of pathogenic ligand-dependent FGFR2 mutations suggests distinct pathophysiological mechanisms for craniofacial and limb abnormalities. Hum Mol Genet 2004;13:2313-2324.

25. Ichida M, Finkel T. Ras regulates NFAT3 activity in cardiac myocytes. J Bio/ Chem 2001;276:3524-3530.

26. Goetz R, Mohammadi M. Exploring mechanisms of FGF signalling through the lens of structural biology. Nat Rev Mol Cell Biol 2013;14:166-180.

27. Sykiotis GP, Plummer L, Hughes VA, et al. Oligogenic basis of isolated gonadotropin-releasing hormone deficiency. Proc Natl Acad Sci USA 2010;107:15140-15144.
28. Bailleul-Forestier I, Gros C, Zenaty D, Bennaceur S, Leger J, de Roux N. Dental agenesis in Kallmann syndrome individuals with FGFR1 mutations. Int J Paediatr Dent 2010;20:305-312.

29. Laitinen EM, Vaaralahti K, Tommiska J, et al. Incidence, phenotypic features and molecular genetics of Kallmann syndrome in Finland. Orphanet J Rare Dis 2011;6:41.

30. Burgar HR, Burns HD, Elsden JL, Lalioti MD, Heath JK. Association of the signaling adaptor FRS2 with fibroblast growth factor receptor 1 (Fgfr 1 ) is mediated by alternative splicing of the juxtamembrane domain. J Biol Chem 2002;277:4018-4023.

31. Twigg SR, Burns HD, Oldridge M, Heath JK, Wilkie AO. Conserved use of a noncanonical 5' splice site (/GA) in alternative splicing by fibroblast growth factor receptors 1, 2 and 3. Hum Mol Genet 1998;7:685-691.

32. Solomon BD, Pineda-Alvarez DE, Mercier S, Raam MS, Odent S, Muenke M. Holoprosencephaly flashcards: A summary for the clinician. Am J Med Genet C Semin Med Genet 2010;154C:3-7.

33. Raivio T, Falardeau J, Dwyer A, et al. Reversal of idiopathic hypogonadotropic hypogonadism. N Engl J Med 2007;357:863-873.

34. Li C, Xu X, Nelson DK, Williams T, Kuehn MR, Deng CX. FGFR1 function at the earliest stages of mouse limb development plays an indispensable role in subsequent autopod morphogenesis. Development 2005;132: 4755-4764.

35. Verheyden JM, Lewandoski M, Deng C, Harfe BD, Sun X. Conditional inactivation of Fgfr1 in mouse defines its role in limb bud establishment, outgrowth and digit patterning. Development 2005;132:4235-4245.

36. van Bokhoven $\mathrm{H}$, Hamel BC, Bamshad M, et al. p63 Gene mutations in eec syndrome, limb-mammary syndrome, and isolated split hand-split foot malformation suggest a genotype-phenotype correlation. Am J Hum Genet 2001;69:481-492.

37. Yang A, Schweitzer R, Sun D, et al. p63 is essential for regenerative proliferation in limb, craniofacial and epithelial development. Nature 1999; 398:714-718.

38. Sun X, Mariani FV, Martin GR. Functions of FGF signalling from the apical ectodermal ridge in limb development. Nature 2002;418:501-508.

39. Ozen RS, Baysal BE, Devlin B, et al. Fine mapping of the split-hand/split-foot locus (SHFM3) at 10q24: evidence for anticipation and segregation distortion. Am J Hum Genet 1999;64:1646-1654.

40. Falardeau J, Chung WC, Beenken A, et al. Decreased FGF8 signaling causes deficiency of gonadotropin-releasing hormone in humans and mice. J Clin Invest 2008;118:2822-2831. 